Abstracta Iranicacta Iranica

Revue bibliographique pour le domaine irano-aryen

Volume 34-35-36 | 2017

Comptes rendus des publications de 2011-2013

\title{
Agnes Korn. Pronouns as Verbs, Verbs as Pronouns: Demonstratives and the Copula in Iranian
}

\section{Agnès Lenepveu-Hotz}

\section{(2) OpenEdition}

10 Journals

\section{Édition électronique}

URL : http://journals.openedition.org/abstractairanica/41113

DOI : 10.4000/abstractairanica.41113

ISSN : 1961-960X

Éditeur :

CNRS (UMR 7528 Mondes iraniens et indiens), Éditions de l'IFRI

Référence électronique

Agnès Lenepveu-Hotz, " Agnes Korn. Pronouns as Verbs, Verbs as Pronouns: Demonstratives and the Copula in Iranian », Abstracta Iranica [En ligne], Volume 34-35-36 | 2017, document 2, mis en ligne le 15 juillet 2016, consulté le 04 octobre 2020. URL : http://journals.openedition.org/abstractairanica/41113 ; DOI : https://doi.org/10.4000/abstractairanica.41113

Ce document a été généré automatiquement le 4 octobre 2020.

Tous droits réservés 


\title{
Agnes Korn. Pronouns as Verbs, Verbs as Pronouns: Demonstratives and the Copula in Iranian
}

\author{
Agnès Lenepveu-Hotz
}

\section{RÉFÉRENCE}

Agnes Korn, « Pronouns as Verbs, Verbs as Pronouns: Demonstratives and the Copula in Iranian ", in: Agnes Korn, Geoffrey Haig, Simin Karimi, Pollet Samvelian, éds., Topics in Iranian Linguistics. Wiesbaden, Reichert, 2011, p. 53-70.

1 Dans cet article, l'A. interroge les cas de conversion de pronominaux en copule ainsi que l'origine de certains clitiques pronominaux, qui proviennent de formes de la copule ou de désinences verbales.

2 Le premier phénomène a déjà été décrit pour les langues iraniennes orientales (sogdien, wakhi, pashto, yaghnobi, ossète), mais l'A. y ajoute une perspective typologique : ainsi on trouve une conversion similaire en chinois, dans des dialectes arabes, en hébreu moderne, etc. Reprenant la théorie d'autres chercheurs, qui voient dans ce phénomène un cas de réanalyse de la construction thème-rhème en une construction sujet-prédicat, l'A. propose aussi d'autres motivations à une telle conversion. L'utilisation de pronoms comme copule pourrait lever des ambiguïtés présentes dans l'ancienne structure de phrase nominale, où la séquence nom-adjectif peut être interprétée comme sujet-prédicat mais aussi comme nom-adjectif épithète. Dans la mesure où phrase nominale et phrase à copule ne sont pas équivalentes (l'A. rappelle l'étude de Benveniste à ce sujet), la stratégie de désambiguïsation a donc consisté à créer une nouvelle copule en utilisant des pronoms. Étant donné que le facteur de l'emprunt a été avancé pour l'hébreu, l'A. se demande si, pour les langues iraniennes aussi, il faut voir un tel facteur : pour le sogdien, on peut ainsi avancer une influence du turc (déjà suggérée par Henning), mais la présence du phénomène dans 
des textes bouddhiques pourrait également suggérer l'existence d'un lien avec le chinois à une époque où shì a encore deux fonctions, l'ancienne de pronom et la nouvelle de copule.

3 Pour ce qui est du cas inverse, certaines langues iraniennes occidentales (balochi, vafsi, nord talyshi, semnani, laki) possèdent des clitiques issus d'anciennes formes de la copule ou de désinences verbales. Ce mécanisme pourrait provenir du fait que les langues iraniennes occidentales ayant de tels clitiques sont aussi caractérisées par la construction ergative. La désinence personnelle qui apparaît pour les verbes intransitifs au présent comme au passé et pour les verbes transitifs au présent a pu être étendue au passé des verbes transitifs, puis réanalysée comme clitique pronominal, justement attendu dans cette structure ergative.

\section{AUTEURS}

\section{AGNÈS LENEPVEU-HOTZ}

Université Sorbonne Nouvelle - Paris 3 